\title{
Wie analysiert man die Lesung eines geschriebenen Gedichts?
}

\section{Monika Rinck liest »Alles Sinnen und Trachten« (Apollo-Theater Siegen, 3. Dezember 2019)}

\section{Jörg Döring}

Zusammenfassung Die Untersuchung versteht sich als Beitrag zu einer empirischen Erforschung von Lyriklesungen und analysiert Monika Rincks Vortrag ihres Gedichtes »Alles Sinnen und Trachten« und die ihn begleitende Rahmungskommunikation auf einer Lesung vom 3. Dezember 2019 in Siegen. Die Ergebnisse dieser Analyse werden in Beziehung gesetzt zu einer traditionell schrifthermeneutischen Lektüre des Gedichts auf der Buchseite.

Schlüsselwörter Empirische Lesungsforschung · Historische Lesungsforschung · Performativer Epitext · Lyriklesungen

\section{How to Analyze the Reading of a Written Poem}

Monika Rinck Reads »Alles Sinnen und Trachten« (Apollo-Theater Siegen, Dezember 3rd 2019)

Abstract The study is intended as a contribution to an empirical study of poetry readings and analyzes Monika Rinck's recital of her poem »Alles Sinnen und Trachten « and the framing communication accompanying it at a reading held in Siegen on December 3, 2019. The results of this analysis are related to a traditional hermeneutic reading of the written form of the poem, published in print.

Keywords Empirical Study of Readings · Historical Study of Readings · Performative Epitext · Poetry Readings

\footnotetext{
J. Döring $(\bowtie)$

Germanistisches Seminar, Universität Siegen, Siegen, Deutschland

E-Mail: doering@germanistik.uni-siegen.de
} 


\section{Historische vs. empirische Lesungsforschung}

Die folgende Untersuchung versteht sich als Beitrag zu einer empirischen Erforschung von Autorenlesungen. Davon gibt es bislang nicht viele in der neueren germanistischen Literaturwissenschaft. Was darauf zurückzuführen sein könnte, dass sie bislang mit der historischen Lesungsforschung alle Hände voll zu tun hatte. ${ }^{1}$ Historische Lesungsforschung ist methodisch anspruchsvoll und stellt hohe Anforderungen an die Quellenkritik, da sie ganz überwiegend anhand schriftlicher Zeugnisse betrieben werden muss, die sich nur vermittelt auf das Lesungsereignis und seine wesentlich oral-auditiven und performativen Anteile beziehen können: »Sie standen dicht um mich herum «, berichtet Friedrich Gottlieb Klopstock 1767 von einer Lesung aus dem Messias in einem Kloster vor 16 Nonnen: »Ich las, und ich sahe nicht wenig Thränen. Ich las fast den ganzen fünften Gesang. Sie verstünden alles, alles, sagten sie; vorher hätten sie nicht alles verstanden. $\ll^{2}$ Das räumliche setting der Lesung (»Sie standen dicht um mich herum «), die setlist des Gelesenen, ein zusammenhängender Werkausschnitt (»den ganzen fünften Gesang «), die Bemühungen des Autors um Resonanzkontrolle im Akt des Vorlesens (»ich sahe nicht wenig Thränen«), dazu ein historisches Publikumsgespräch, erschütterte Nonnen, hermeneutische Präsenzeffekte: Über all das gibt diese Quelle Auskunft. Wie gern wär' man doch als Lesungsforscher bei dieser Dichterlesung dabei gewesen! So aber sind wir auf die gewiss nicht unparteiliche Retrospektion eines entweder stolzen Autors angewiesen, dessen Vorleseperformanz das hier bekundete Totalverstehen (»alles, alles«) erst stimuliert habe. Oder geht Klopstock hier vielmehr im ironischen Berichtskonjunktiv auch auf auktoriale Distanz zu der Emphase seiner Zuhörerinnen (»Sie verstünden alles, alles, sagten sie; vorher hätten sie nicht alles verstanden $\ll)$ ?

Die historische Lesungsforschung hat des Weiteren mit dem Umstand zu kämpfen, dass auch in den Tonarchiven seit der Erfindung technischer Medien zur Aufzeichnung der menschlichen Stimme (zunächst mit Hilfe des Phonographen von 1889) bis weit ins 20. Jahrhundert hinein sich nur »eine recht kümmerliche Liste« von Aufnahmen deutschsprachiger SchriftstellerInnen erhalten hat:

\footnotetext{
${ }^{1}$ Ein epochales, zweibändiges Werk zur Geschichte der literarischen Vortragskunst ist - nach jahrzehntelanger Forschungstätigkeit - jetzt endlich erschienen: Meyer-Kalkus, Reinhart: Geschichte der literarischen Vortragskunst. Stuttgart 2020 (2 Bd.). Die Autorenlesung ist darin ein gewichtiger Fall unter anderen. Vgl. dazu überdies: Maye, Harun: Die Entstehung der Dichterlesung. In: Natalie Binczek/Uwe Wirth (Hg.): Handbuch Literatur \& Audiokultur. Berlin/New York 2020, S. 307-317; ders.: Literatur aus der Sprechmaschine. Zur Mediengeschichte der Dichterlesung von Klopstock bis Rilke. In: Natalie Binczek/ Cornelia Epping-Jäger (Hg.): Das Hörbuch. Audioliteralität und akustische Literatur. München 2014, S. 13-29; ders.: Eine kurze Geschichte der Dichterlesung. In: Sprache und Literatur 110 (2012), S. 38-49; Grimm, Gunter E.: Nichts ist widerlicher als eine sogenannte Dichterlesung. Deutsche Autorenlesungen zwischen Marketing und Selbstpräsentation. In: Ders./Christian Schärf (Hg.): Schriftsteller-Inszenierungen. Bielefeld 2008, S. 141-167; Müller, Lothar: Die zweite Stimme. Vortragskunst von Goethe bis Kafka. Berlin 2007; Böhm, Thomas: Lesung. In: Erhard Schütz et al. (Hg.): Das BuchMarktBuch. Der Literaturbetrieb in Grundbegriffen. Reinbek b. Hamburg 2005, S. 203-206; Göttert, Karl-Heinz: Geschichte der Stimme. München 1998; Tghart, Reinhard (Hg.): Dichter Lesen (3 Bd.) Marbach 1984-1995.

2 Klopstock brieflich an den Jesuitenpater und Ossian-Übersetzer Johann Michael Denis am 6. Januar 1767. Zit. nach Maye 2020, S. 313.
} 
Felix Dahn 1899, Marie von Ebner-Eschenbach 1901, Ferdinand von Saar 1901, Ernst von Wolzogen 1904, Arthur Schnitzler 1907, Hugo von Hofmannsthal 1907, Gerhart Hauptmann 1922, Joachim Ringelnatz 1922, Hans Friedrich Blunck 1927. Nur neun Stimmen bekannter Schriftsteller haben in den 40 Jahren zwischen 1889 und 1927 dauerhafte Spuren hinterlassen. ${ }^{3}$

Nicht viel besser ist die Quellenlage für die Zeit danach. Seit Ende der 1920er Jahre begann der Hörfunk damit, Dichterlesungen (wie solche von Thomas Mann, Gottfried Benn oder Karl Kraus) auf Schellackplatten mitzuschneiden, und private Schallplattenfirmen versuchten, diese Aufnahmen zu vermarkten (Abspielzeit: maximal vier Minuten). Dennoch bleibt die Archivbilanz enttäuschend: »Das Erhaltengebliebene ist zufällig hinsichtlich der Umstände seiner Überlieferung wie auch hinsichtlich der Auswahl der auf Schallplatte aufgezeichneten Autoren und Texte. $\aleph^{4}$ Insbesondere dann enttäuscht die Archivbilanz, wenn man sich für Praxis von Autorenlesungen vor Publikum interessiert und nicht nur für die literarische Vortragskunst unter Studiobedingungen. Letztere ist seit der Durchsetzung des öffentlich-rechtlichen Rundfunks nach 1948 in Westdeutschland recht gut überliefert. Das Format der Autorenlesung im Radio hat sich im Prinzip bis heute erhalten und leistet wegen der vergleichsweise guten Honorierung (und nicht zuletzt aufgrund der kurativen Kollaboration von Autorkollegen in den Rundfunkredaktionen, die der Literatur Gewicht und Sendeplatz einräumten) einen wichtigen Beitrag zur Existenzsicherung von Autorinnen und Autoren. ${ }^{5}$

Aber gerade weil die Bedingungen für studioproduzierte Autorenlesungen in der zweiten Hälfte des 20. Jahrhunderts so exzeptionell günstig waren, finden sich in den Radioarchiven relativ wenig Live-Mitschnitte historischer Literaturlesungen vor Publikum. Was zeitgleich in Buchhandlungen, Kulturhäusern und Schulklassen als Teil der (immer öfter vitalistisch »literarisches Leben « genannten) Öffentlichkeit ${ }^{6}$ an Lesungspraxis vor Ort und in der Fläche sich ereignete, ist schlecht dokumentiert, vor allem was die Verfügbarkeit von Audio-Quellen anbetrifft. Daran hat sich auch nichts geändert, als in den 1960er Jahren das Fernsehen sich als Leitmedium der westdeutschen Öffentlichkeit etablierte und die Ton- und Bildspeicher sich rasch auch mit Originalaufnahmen deutschsprachiger SchriftstellerInnen zu füllen begannen. Historische Lesungspraxis selbst ist darin allenfalls als illustrativ-atmosphärischer Ausschnitt überliefert ${ }^{7}$, was auch im Allgemeinen strukturbildend ist

\footnotetext{
3 Meyer-Kalkus, Reinhart: Akteure, Formate und Medien der literarischen Vortragskunst. In: Natalie Binczek/Uwe Wirth (Hg.): Handbuch Literatur \& Audiokultur. Berlin/New York 2020, S. 318-344, hier: S. 335.

4 Ebd., S. 336.

5 Vgl. Boehncke, Heiner/Michael Crone (Hg.): Radio Radio. Studien zum Verhältnis von Literatur und Rundfunk. Frankfurt a. M. 2005.

${ }^{6}$ Lorenz, Otto: Literarisches Leben. In: Harald Fricke et al. (Hg.): Reallexikon der deutschen Literaturwissenschaft. Bd. II. Berlin/New York 2007, S. 438-441.

7 Vgl. die sechsteilige TV-Dokumentation zur Geschichte der Nachkriegsliteratur, die der Literaturwissenschaftler Volker Bohn daraus 1993 für das ZDF/3sat zusammengestellt hat: Deutsche Literatur seit 1945 - Nachrichten von Büchern und Menschen (zus. 270 min).
} 
für die Ton- und Bildspeicher des TV im Unterschied zu denen des Radios. ${ }^{8}$ Und die für die Geschichte der westdeutschen Nachkriegsliteratur so folgenreiche Praxis der Autorenlesungen bei den Treffen der Gruppe 47 hatte zwar schon bald ihren Werkstattcharakter eingebüßt und fand unter immer größer werdender Beteiligung der literarischen Öffentlichkeit statt. Das bezieht sich vor allem auf die Beteiligung von Literaturkritikern und VerlagsvertreterInnen an den Gruppendiskussionen und die dazugehörige Print-Berichterstattung. Audiomitschnitte von der Gruppe 47 gibt es fast keine - mit Ausnahme der legendären Auslandstagung in Princeton 1966, die vor allem für die Beschimpfungssuada von Peter Handke berühmt ist, mit der in vieler Hinsicht auch der Anfang vom Ende der Gruppe sich ankündigte. ${ }^{9}$ Besser dokumentiert - d.h. auch in Form von Bild/Ton-Aufzeichnung der gesamten Lesungspraxis - ist der Klagenfurter Wettbewerb um den Ingeborg-Bachmann-Preis, der seit 1989 in voller Länge vom Fernsehsender 3sat übertragen wird, aber aufgrund seiner sehr besonderen Spielregeln eher für Praktiken des Kritikergesprächs dokumentarisch einschlägig ist als für den ordinary case der Lesungsforschung: die so genannte > Wasserglaslesung « von AutorInnen vor Publikum - die trotz der viel beschriebenen Eventisierung des Literaturbetriebs seit den 1990er Jahren ${ }^{10}$ nach wie vor den am weitesten verbreiteten Typ von öffentlicher Autorenlesung darstellt. ${ }^{11}$

Die Quellenlage dazu hat sich erst mit der Popularisierung des Internet seit Mitte der 1990er Jahre und der massenhaften Verfügbarkeit von preiswerter, dennoch qualitativ hochwertiger und von Laien bedienbarer Medientechnologie zur Bild-/ Tonaufzeichnung auf dem Konsumgütermarkt entscheidend verbessert. Seither werden immer mehr Lesungen mitgeschnitten (von VeranstalterInnen, VerlegerInnen, AutorInnen, teils von den Zuhörenden) und verstärkt auch das Internet als Speicher- und Zirkulationsort solcher Dokumentationen genutzt. Auf diese Materialbasis kann eine Forschung zurückgreifen, die zeitgenössische Lesungspraktiken jetzt auch empirisch $\mathrm{zu}$ untersuchen versucht. ${ }^{12}$ Eine solche Forschung interessiert sich für die Lesung als integrale Praxisform, die AutorInnen, Texte und Publikum für eine begrenzte Zeit räumlich und situativ vergemeinschaftet und die bei der typischen >Wasserglaslesung < vor allem durch mündlichen Werkvortrag der AutorInnen strukturiert wird. Dabei handelt es sich zumeist um die Reoralisierung einer zuvor schriftlich fixierten Textgrundlage - gleichsam »als Verfahren einer Renaturalisie-

\footnotetext{
${ }^{8}$ Döring, Jörg: Fernsehen. In: Erhard Schütz et al.: Das BuchMarktBuch (s. Anm. 1), S. 121-125. Und ders.: Der Autor im Fernsehen. In: Erhard Schütz/Thomas Wegmann: literatur.com. Tendenzen im Literaturmarketing. Berlin 2002, S. 137-171.

9 Vgl. Wegmann, Nikolaus/Reiber, Cornelius: Deutsche Literatur. Die Gruppe 47 in Princeton. In: Sprache und Literatur 110 (2012), S. 50-65; Jörg Döring: Peter Handke beschimpft die Gruppe 47. Siegen 2019.

10 Vgl. Porombka, Stephan: Slam, Pop und Posse. Literatur in der Eventkultur. In: Matthias Harder (Hg.): Bestandsaufnahmen. Deutschsprachige Literatur der neunziger Jahre aus interkultureller Perspektive. Würzburg 2001, S. 27-42; ders.: Schriftstellerberuf. In: Thomas Anz (Hg.): Handbuch Literaturwissenschaft. Bd. 3: Institutionen und Praxisfelder. Stuttgart/Weimar 2007, S. 283-294.

11 Vgl. Geulen, Benedict: Wasserglaslesungen sind die Regel. In: Buchreport v. 8. April 2010; Johannsen, Anja: »Stroh zu Gold oder Gold zu Stroh?« Zur Ambivalenz öffentlicher Autorenlesungen. In: Philipp Theisohn/Christine Weder (Hg.): Literaturbetrieb. Zur Poetik einer Produktionsgemeinschaft. Paderborn 2013, S. 63-76.

12 Döring, Jörg/Paßmann, Johannes: Lyrik auf YouTube. Clemens J. Setz liest »Die Nordsee« (2014). In: Zeitschrift für Germanistik N.F. XXVII 2 (2017), S. 329-347.
} 
rung des Geschriebenen, als Rückgewinnung der ursprünglichen Werkschöpfung und als sprachangemessene Verbindung von Wort und Schrift. « ${ }^{13}$ Es hängt von der Güte der Aufzeichnung ab, inwieweit empirische Lesungsforschung alle Aspekte dieser Praxisform gleichrangig zu ihrem Gegenstand machen kann. Um das Autorhandeln vor Publikum und die situative Kommunikation mit dem Publikum zu untersuchen, reicht i.d.R. eine gute Tonaufnahme (auch im vorliegenden Fall haben wir ganz bewusst auf eine Videoaufzeichnung verzichtet, um die Dokumentation der Praxis weniger invasiv zu gestalten). Um darüber hinaus auch die Hörerfahrungen, Verstehensleistungen und die nonverbale Resonanz des Publikums zu erfassen, bedarf es zusätzlicher empirischer Begleitforschung in Form von Auswertungsgesprächen und Fragebögen. ${ }^{14}$ In der folgenden Untersuchung einer Lesung der Lyrikerin Monika Rinck am 3. Dezember 2019 im Siegener Apollo-Theater steht aus Umfangsgründen vor allem das Autorinnenhandeln im Mittelpunkt: Welche Kommunikation geht mit dem für eine Lyriklesung konstitutiven Werkvortrag einher und rahmt diesen? Und mit welchem Effekt? ${ }^{15}$

\section{Die Lyriklesung: Werkvortrag und performativer Epitext}

Die Lyriklesung stellt einen Sonderfall unter den zeitgenössischen Autorenlesungen vor Publikum dar. Anders als bei den Lesungen von Prosa, i.d.R. aus neu erschienenen Romanen, werden bei Lyriklesungen nicht lediglich ausgewählte Werkausschnitte vorgetragen, sondern ganze Werke, und diese in loser Serie. Theatertexte sind kaum Gegenstand von Autorenlesungen, einfach weil sie als Werkmedien zweiter Ordnung eine Aufführungspraxis eigener Art nach sich ziehen. Prosalesungen indes folgen zumeist der Novitätenlogik des saisonal strukturierten Buchmarkts. Sehr selten, dass in einer Prosalesung mehr als nur Ausschnitte aus dem jeweils aktuellen Roman vorgetragen werden. Anlass einer Lyriklesung mag auch ein neu erschienener Gedichtband sein, aber strukturell stellt der Gedichtband selbst bereits eine Kompilation separater Werke dar, aus der für den Vortrag bei einer Autorenlesung einzelne ausgewählt werden, die aber wiederum in aller Regel vollständig vorgetragen werden. Gar nicht so selten überdies, dass bei Lyriklesungen die Novitätenlogik des Buchmarkts durchbrochen wird und auch Gedichte früherer Schaffensperioden oder Veröffentlichungen der Autorin oder des Autors vorgetragen werden. Insofern

\footnotetext{
13 Möllendorf, Peter v.: Vortrag/Lesung: Begriffsexplikation. In: Natalie Binczek/Till Dembeck/Jörgen Schäfer (Hg.): Handbuch Medien der Literatur. Berlin/Boston 2013, S. 333.

14 Vgl. Utler, Anja: «manchmal sehr mitreißend«. Über die poetische Erfahrung gesprochener Gedichte. Bielefeld 2016. Solche empirische Forschung zu den Hörerfahrungen des Publikums bei Lesungen wäre zu unterscheiden von der empirisch-kognitivistischen Rezeptionsforschung, die nur unter Laborbedingungen durchgeführt wird. Vgl. z.B. Menninghaus, Winfried et al.: The emotional and aesthetic powers of parallelistic diction. In: Poetics 63 (2017), S. 47-59.

15 Nur sehr kursorisch sollen hier auch die Ergebnisse einer Publikumsbefragung miteinfließen, die während dieser Lesung von unserer Forschungsgruppe ebenfalls durchgeführt wurde. Die Untersuchung insgesamt erfolgte im Rahmen des FWF/DFG-Projekts »Formen und Funktionen auktorialer Epitexte im literarischen Feld der Gegenwart« (Universität Innsbruck/Universität Siegen). Vgl. https://blogs.uni-siegen. de/epitexte/ (09.01.2021).
} 
können Lyriklesungen zur auktorial kuratierten Werkschau tendieren, in der eine anlassgegebene Auswahl von Gedichten aus dem Gesamtwerk zusammengestellt wird (darin strukturell dem Gedichtband als Werkmedium verwandt).

Dabei ist der Status der Gedichtlesung als Werkmedium gar nicht so leicht $\mathrm{zu}$ bestimmen: Einerseits wird die Lesung eines Gedichts gegenüber seiner »textförmig erscheinenden« Vorlage »üblicherweise als nachträglich angesehen « ${ }^{16}$, als Oralisierung eines schriftlich fixierten Textes, der in der Performanz seines Vortrags an Stimme und Körper seiner Urheberin oder seines Urhebers rückgebunden wird. Andererseits kommt der Lesung ihrerseits ein transitorischer Werkcharakter zu, insofern das Gedicht vollständig vorgetragen wird und rezeptiv gar nicht unbedingt der vorgängigen Lektüre des schriftlich fixierten Gedichtes bedarf. (Vielleicht ist das gar eine Tendenz rezenter Lyrikrezeption: die Lesung als autonome Aufführungspraxis anzusehen, die »nicht mehr als Anhängsel zum gedruckten Buch bestimmt werden kann $\ll .{ }^{17}$ Von den 58 Besucherinnen und Besucher der hier ausgewerteten Monika Rinck-Lesung von 2019 gaben mehr als zwei Drittel an, mit dem Werk der Autorin vorgängig nicht vertraut gewesen zu sein.)

Darf man eine Gedichtlesung daher überhaupt mit den paratexttheoretischen Begriffen Gérard Genettes beschreiben? Sie ist ersichtlich mehr als »Beiwerk« des Buches $^{18}$, sie geht nicht in öffentlich geäußertem auktorialem »Epitext « auf. Der Vortrag des Gedichts hat seinen Ort - in der räumlichen Logik des Paratextkonzepts - keinesfalls »anywhere out of the book «19 (im Unterschied zu dem an den Buchkörper gebundenen Peritext wie Autorname, Überschrift und Untertitel...), sondern tritt auf Zeit als eigenständiges, vorgetragenes Werk unabhängig vom Buch vor die räumlich und situativ als Lesungspublikum vergemeinschaftete literarische Partialöffentlichkeit. Das gilt wohlgemerkt nur für den Gedichtvortrag selbst. Zeitgenössische Lyriklesungen bestehen aber in den seltensten Fällen nur aus Gedichtvortrag. Begleitet wird der auktoriale Werkvortrag durch eine lesungstypische Form der Rahmungskommunikation. Eine Moderation stellt den Autor oder die Autorin anfangs vor, führt evtl. während oder nach der Lesung auch noch ein Gespräch über das Werk, vielleicht gibt es am Ende ein Publikumsgespräch, in welchem die Zuhörenden dem Autor oder der Autorin Fragen stellen oder mit ihm/ihr diskutieren. Feinsegmentiert wird die Serie von Gedichtvorträgen des Weiteren durch ein- oder ausleitende spontanmündliche Kommentare der Autorin oder des Autors. ${ }^{20}$ Als Rahmungskommunikation dienen sie lesungspragmatisch vor allem dazu, Werkgrenzen hörbar zu machen: sie fungieren als Trenner und markieren auditiv (für ein $\mathrm{Pu}-$ blikum, das die Gedichte in aller Regel nicht mitlesen kann, sofern es nicht doch

\footnotetext{
16 Maye, Harun: Vortrag/Lesung. In: Natalie Binczek/Till Dembeck/Jörgen Schäfer (Hg.): Handbuch Medien der Literatur. Berlin/Boston 2013, S. 341.

17 Ebd.

18 Genette, Gérard: Paratexte. Das Buch vom Beiwerk des Buches. Frankfurt a. M. 2001, S. 10.

19 Ebd., S. 328.

20 Dass sie nicht immer ganz spontan sind, sondern - sofern sie sich kommunikativ bewährt haben - auch Teil einer Epitext-Routine werden können, zeigt sich, wenn man mehrere Lesungen eines Autors, einer Autorin miteinander vergleicht. Vgl. Döring, Jörg: Marcel Beyer liest. Gedicht und performativer Epitext. In: Christian Klein (Hg.): Marcel Beyer. Perspektiven auf Autor und Werk. Stuttgart 2018, S. 73-93.
} 
einen Gedichtband mitgebracht hat...), wo ein Gedicht beginnt und wo es endet. Für die Zuhörenden eröffnen diese auktorialen Kurzkommentare z.B. einen Hallraum für die Nachwirkung eines soeben gehörten Gedichts, ohne dass dieser nur mit andächtigem Schweigen gefüllt wäre. Die Rahmungskommunikation zerdehnt künstlich die Zeit zwischen zwei Werkvorträgen (vergleichbar der Zeit, die beim Gang von Bild zu Bild in der Kunstausstellung vergeht) und nimmt so Rücksicht auf die ästhetische Verarbeitungskapazität der Zuhörenden. Auf jeden Fall stellen sie überdies auch auktoriale Kurzkommentare zum Werkvortrag dar: Hinweise zur Textentstehung werden gegeben, Erläuterungen zum Verständnis, Hörvorschriften und Interpretamente zu einer möglichen Deutung des soeben Gehörten oder des gleich noch zu Hörenden. Für die inszenierte Präsenz des Werks in der Lesung, verkörpert durch die Autorstimme, erscheinen diese auktorialen Kurzkommentare gleichsam als Begleitschutz und Schmiermittel. Kaum eine Lyriklesung verzichtet darauf. Mehr noch: Zeitlich übersteigt die Dauer dieser Rahmungskommunikation die Dauer des eigentlichen Gedichtvortrags nicht selten beträchtlich. Sie erst scheint die rezeptive Verstehens->Zumutung<, die darin besteht, ein womöglich unbekanntes Gedicht nur einmal zu hören (ohne die Möglichkeit einer vertiefenden Re-Lektüre wie bei der Buchrezeption), ästhetisch kommensurabel zu machen. (Die hier ausgewertete Lesung von Monika Rinck im Siegener Apollo Theater 2019 dauerte insgesamt eine Stunde und 26 Minuten. Davon entfielen nicht mehr als 27 Minuten auf den reinen Gedichtvortrag. Der Rest war Rahmungskommunikation im beschriebenen Sinne. Darf man hier überhaupt noch von Rahmungskommunikation sprechen...?)

Diese spontanmündlichen auktorialen Kurzkommentare bei Lesungen - wohlgemerkt: nicht der Gedichtvortrag selbst - sollen hier in Erweiterung des Genetteschen Begriffsinventars als performativer Epitext bezeichnet werden. ${ }^{21}$ Sie sind semi-öffentlicher Epitext, insofern sie sich auf das Werk beziehen, es kommentieren (so wie in Autoreninterviews, öffentlichen Antworten auf Kritiken, Kolloquien, Debatten oder Poetikvorlesungen), aber vom Werkvortrag klar getrennt sind. Sie sind performativ, insofern sie als Selbstkommentare der Autorin oder des Autors auf ihren situativen Kontext verweisen und als integraler Bestandteil der Praxis einer Wasserglaslesung die transitorische Performanz des Gedichts erst mithervorbringen. Dazu gibt es bislang erstaunlich wenig empirische deutschsprachige Forschung. ${ }^{22}$

\footnotetext{
21 Vgl. auch Döring, Jörg: Marcel Beyer liest; Manz, Nora: Lyrik und performativer Epitext: Nora Gomringers Lesungsroutinen. In: Zeitschrift für Literaturwissenschaft und Linguistik 49 (2019) H. 3, S. 477-492.

22 Die vorliegende Forschung bezieht sich bislang eher auf spektakulärere, tendenziell theatralische Formen der Lyrik-Performanz wie Slam, Spoken Word oder Lyrik-Performances. Vgl. Benthien, Claudia/ Prange, Catrin: Spoken-Word-Literatur und Poetry Slam. In: Natalie Binczek/Uwe Wirth (Hg.): Handbuch Literatur \& Audiokultur. Berlin/New York 2020, S. 307-317; Novak, Julia: Live-Lyrik. Körperbedeutung und Performativität in Lyrik-Performances. In: Anna Bers/Peer Trilcke (Hg.): Phänomene des Performativen in der Lyrik. in der Lyrik. Systematische Entwürfe und historische Fallbeispiele. Göttingen 2017, S. $147-162$.
} 


\section{3 »What it is that only silent reading of the words on the page can achieve for a reader of the poem? $\ll^{23}$}

Etwas anders sieht es erfreulicherweise in der angelsächsischen Forschung aus, die sich schon seit längerem auch für die Aufführungsbedingungen von Gedichten und für Lesungspraktiken von Autorinnen und Autoren interessiert ${ }^{24}$ - vielleicht weil in Großbritannien und den USA die Bedeutung von Gedichten nicht nur für das literarische, sondern auch für das öffentliche Leben immer noch viel größer ist als im deutschsprachigen Raum. (Oder können Sie sich vorstellen, dass hierzulande eine 22-jährige Nachwuchs-Lyrikerin bei der Inauguration des Bundespräsidenten ein Widmungsgedicht vortragen dürfte? Oder Nora Gomringer als poeta laureata die neue Legislaturperiode des deutschen Bundestags einleitete? $)^{25}$

Die hier vorgelegte Untersuchung orientiert sich am Design einer für empirische Lesungsforschung maßgeblichen Studie, die der englische Literaturwissenschaftler Peter Middleton schon 2005 durchgeführt hat: »How to Read a Reading of a Written Poem«. Darin analysiert er u.a. Jackie Kays Lesung ihres Gedichts »Brendon Gallacher « von 1998. ${ }^{26}$ Wie schon der Titel des Aufsatzes verrät, geht auch Middleton von der Vorgängigkeit des geschriebenen Gedichts gegenüber der Aufführungssituation aus. Das heißt aber nicht, dass in der Autorinnen-Lesung das Gedicht nicht situativ anderes bedeutet, als wenn es in zerdehnter Kommunikation (d.h. ohne die Anwesenheit der Autorin) allein im stillen Kämmerlein oder in der Interpretationsgemeinschaft in Universitätsseminar bzw. Klassenzimmer gelesen (und wieder gelesen) wird. Damit vermeidet Middleton auf sympathisch-pragmatische Weise sowohl den Extremismus der hermeneutischen Schriftgläubigkeit, die in der Lesung nur nachgeordnetes und vergängliches Beiwerk der ehernen Textform erkennen kann (vielleicht gibt es deshalb in der germanistischen Literaturwissenschaft so viel Gedichtexegese und kaum Lesungsforschung...); als auch zeigt er sich unempfänglich für das Geraune mancher Essays aus den Performance Studies, die - anstatt eine

\footnotetext{
23 Middleton, Peter: How to Read a Reading of a Written Poem. In: Oral Tradition 20 (2005), H. 1, S. 7-34, hier: S. 10.

24 Vgl. u.a. Gräbner, Cornelia: Poetry and Performance: The Mersey Poets, The International Poetry Incarnation and Performance Poetry. In: Edward Larissy (Hg.): The Cambridge companion to British poetry since 1945. Cambridge 2015, S. 68-81; Foley, John Miles: How to read an Oral Poem. Urbana 2002; Middleton, Peter: Poetry's Oral Stage. In: Ivan Gaskell/Salim Kemal (Hg.): Performance and Authenticity in the Arts. Cambridge 1999, S. 154-186; Bernstein, Charles: Close Listening: Poetry and the Performed Word. New York 1998. Middleton, Peter: The Contemporary Poetry Reading. In: Charles Bernstein (Hg.): Close Listening: Poetry and the Performed Word. New York 1998, S. 262-299.

25 Doch vielleicht ist es bald so weit. Nora Gomringer selbst schrieb dazu am 20. Januar 2021 den folgenden Facebook-Post: »Inaugural Poetry - it is somewhat of a genre! When I proposed something like this for Germany in the midst of Lockdown one (March/April), minister Dorothee Bär was open to the idea to have poets at the Bundestag opening sessions. I really believe : politics DO benefit from poetic wit \& wisdom, insight and aura. I really do.« Vielen Dank an Nora Manz für die Mitteilung dieses Fundstücks.

26 Ein Video dieser Lesung mit dem dazu gehörigen performativen Epitext der Autorin kann man heute noch auf YouTube finden: https://www.youtube.com/watch?v=NXJGwsmmEaQ (letzter Zugriff: 21.1.2021).
} 
Aufführung zu analysieren - viel lieber deren Singularität, Unwiederholbarkeit und epiphanische Ereignishaftigkeit beschwören. ${ }^{27}$

Für den pragmatischen Lyriklesungsforscher Middleton gilt vielmehr: »Both silent reading and oral performance are incomplete scenes of reception. $«{ }^{28}$ Das Gedicht »requires both to be read on the page and to be heard aloud by the poet, because these poems extend over time and memory to create effects that depend on a mutual interdependence of performance and silent interpretation. $\ll^{29}$ Methodisch hat das zur Folge, dass Middleton zunächst mit der Lektüre der Schriftform des Gedichtes beginnt: »What is it that only silent reading of the words on the page can achieve for a reader of the poem? $\ll^{30}$, um danach die hermeneutische Differenz zu der Lektüre des Gedichtes als auktorial vorgetragenes (und epitextuell gerahmtes) zu bestimmen. Beide Lektüren sind nicht fundamental wesens- oder wertverschieden, sondern verweisen aufeinander, vervollständigen sich idealerweise wechselseitig (so die fast literaturpädagogisch anmutende Prämisse Middletons, der beides gleichrangig für wertvoll erachtet: die stille Rezeption des Gedichts auf der Buchseite wie den auktorialen Gedichtvortrag bei einer Lyriklesung).

Dieser Zweischritt der Analyse soll hier am Beispiel des Gedichts »Alles Sinnen und Trachten« von Monika Rinck nachvollzogen werden: zunächst a) eine (kursorische) Lektüre der Schriftform des Gedichts, so wie es sich zuerst in dem Gedichtband Helle Verwirrung von 2009 findet. ${ }^{31}$ Sodann soll analysiert werden, wie b) Monika Rinck dieses Gedicht bei einer Lesung im Siegener Apollo-Theater am 3. Dezember 2019 epitextuell ankündigt und dann vorträgt. a) ist gleichsam der ordinary case literaturwissenschaftlicher Beschäftigung mit Lyrik; b) läuft auf die Untersuchung von performativem Epitext und Werkvortrag in einer monologischen Gesprächssituation hinaus und muss daher - neben den Methoden der performance studies zur Analyse von Aufführungsereignissen - auch Methoden zur Analyse gesprochener Sprache aus der linguistischen Pragmatik in Anspruch nehmen.

\section{ALLES SINNEN UND TRACHTEN}

V.1 die menschen waren schlechte dichter

V.2 und ihre töchter trugen riesige bastarde aus.

V.3 das ist das ende, beschied gott im himmel.

V.4 und kein fünkchen güte erhellte den pechschwarzen uferwall.

V.5 die wasser stürzten.

\footnotetext{
27 Vgl. z. B. Sätze wie dieser: »Aufführungen [...] erschöpfen sich in ihrer Gegenwärtigkeit, d. h. ihrem dauernden Werden und Vergehen, in ihrer Autopoiesis.«. In: Erika Fischer-Lichte: Einleitende Thesen zum Aufführungsbegriff. In: Dies./Clemens Risi/Jens Roselt (Hg.): Kunst der Aufführung - Aufführung der Kunst. Berlin 2004, S. 11-26, hier: S. 14.

28 Middleton: How to Read a Reading, S. 9.

29 Ebd.

30 Ebd., S. 10.

31 Rinck, Monika: Alles Sinnen und Trachten. In: Dies.: Helle Verwirrung. Gedichte. Idstein 2009, S. 69. Wiederabgedruckt wurde es mittlerweile in (wiederum als Schlussgedicht): Monika Rinck: Champagner für die Pferde. Ein Lesebuch. Hg. v. Monika Rinck u. Daniela Seel. Frankfurt am Main 2019, S. 508.
} 
V.6 (und manch einer sagte sich:

V.7 bevor ich aus allen tieren

V.8 paare bilde und verlade,

V.9 geh ich lieber unter. $)^{32}$

Die Gedichtüberschrift zitiert einen berüchtigten Vers aus der hebräischen Bibel, 1. Buch Mose $6.5 \mathrm{ff}$, den Beginn der Sintflutgeschichte, Gottes Urteil über die verdorbene Menschheit, die Reue über sein Schöpfungswerk und den daraus abgeleiteten Vernichtungsbeschluss: »Der Herr sah, daß auf der Erde die Schlechtigkeit des Menschen zunahm und daß alles Sinnen und Trachten seines Herzens immer nur böse war. Da reute es den Herrn, auf der Erde den Menschen gemacht zu haben, und es tat seinem Herzen weh. Der Herr sagte: Ich will den Menschen, den ich erschaffen habe, vom Erdboden vertilgen, mit ihm auch das Vieh, die Kriechtiere und die Vögel des Himmels [...] «33 Dadurch, dass die Gedichtüberschrift bei Rinck den moralischen Furor des Bibelverses abschneidet, bleiben Subjekt wie Objekt des Sinnens und Trachtens vorerst unbestimmt, richtet sich die Wahrnehmung allein auf zwei freigestellte, altertümliche, eine Denkarbeit bezeichnende Tätigkeitsworte und ihren Klang. Ohne das göttliche Verdammungsurteil sind »Sinnen und Trachten« nurmehr sanfte daktylische Wellen (mit zwei freischwebenden Betonungen als Auftakt).

Es klingt zudem, als seien die beiden zweisilbigen Tätigkeitsworte durch Binnenreim aneinander gebunden, unauflösbar auch semantisch, weil immer unklarer wird, was »Sinnen « (»seine gedanken worauf richten, nachdenken $\left.\aleph^{34}\right)$ und $\gg$ Trachten« (»überlegen«, sagt das Grimmsche Wörterbuch ${ }^{35}$ ) eigentlich voneinander unterscheidet. Wer Monika Rincks Zyklus »Der Quitte wegen«, mit dem sie den Band Helle Verwirrung eröffnet, und ihre Lust an Klangsemantik zwischen den Sprachen kennt (»Komet Quitte/Quit: to quit him oder her, aber wenn, dann in anger ${ }^{36}$ ), der mag bei »Sinnen« auch das englische $\sin$ schon heraushören. Noch sind es sanfte daktylische Wellen, auf denen das Klangschiff dieser biblischen Kopula schaukelt, aber die Sintflut kündigt sich schon an.

Der erste Vers des Gedichts spezifiziert dann den göttlichen Verdammungsgrund auf überraschende Weise: »die menschen waren schlechte dichter«. Nicht die Bosheit ihrer Herzen ist bestrafungswürdig, sondern dass sie - in den Ohren des Gottes - minderwertige Verse machen. Das Versemachen scheint eine verdammt ernste Angelegenheit zu sein. Wird das Gedicht hier auf größenwahnsinnige Weise selbst-

\footnotetext{
32 Die vollständige Wiedergabe des Gedichts erfolgt mit freundlicher Erlaubnis von Daniela Seel, kookbooks Verlag, heute Berlin.

33 Zit. n. Neue Jerusalemer Bibel. Einheitsübersetzung. Mit dem Kommentar der Jerusalemer Bibel. Freiburg/Basel/Wien 1985, S. $21 \mathrm{f}$.

34 Deutsches Wörterbuch von Jacob Grimm und Wilhelm Grimm, Berlin-Brandenburgische Akademie der Wissenschaften, Bd. 16, Sp. 1165. In: https://woerterbuchnetz.de/?sigle=DWB\#1 (zuletzt abgerufen: 30.01.2021).

35 Ebd., Bd. 21, Sp. 995. In: https://woerterbuchnetz.de/?sigle=DWB\#1 (zuletzt abgerufen: 30.01.2021).

36 Rinck, Helle Verwirrung, S. 18. »Die Quitte wird ihr zur Metapher des Endes, weil in ihr das englische to quit ruht «, so sehr prägnant Christian Metz in der bislang besten Analyse zum Werk Monika Rincks. In: Christian Metz: Poetisch denken. Die Lyrik der Gegenwart. Frankfurt am Main 2018, S. 138.
} 
referentiell? Dichter verdienen Vernichtung, weil sie schlecht geliefert haben? Ein Blick auf den ursprünglichen Wortlaut in der Luther-Bibel verrät, dass die Engführung von Sünde und Dichten hier schon grundgelegt ist: »Als aber der HERR sah, daß der Menschen Bosheit groß war und alles Dichten und Trachten ihres Herzens nur böse war immerdar, da reute es ihn, daß er die Menschen gemacht hatte auf Erden $[\ldots] \ll^{37}$ Alles »Sinnen« war früher ein »Dichten«, und deshalb will der Gott keinesfalls nur die Dichter richten, sondern alle Menschen als »schlechte dichter «. Indem das Gedicht mithin eine ältere Bedeutungsvariante von »Sinnen« poetisch restituiert, macht es Gebrauch von einem wichtigen poetologischen Grundsatz, den Monika Rinck in ihrer lyrikline-Festrede 2009 formuliert hat: »Erinnern wir uns daran, dass in den Begriffen, mit denen wir heute täglich umgehen, immer auch ein Erfahrungsrest ihres früheren Gebrauchs sedimentiert ist [...] Begriffsgeschichte ist Konfliktgeschichte. Wer Worte sorgsam setzt, hat damit zu tun. $\ll^{38}$

Auch der Folgevers 2 »und ihre töchter trugen riesige bastarde aus « lässt sich auf den Kontext der Noah-Geschichte beziehen, allerdings auf einen Teil, der in Sonntagspredigt oder Kinderbibelillustration zur Sintflut eher weniger thematisch ist: unmittelbar, bevor der Gott seinen Tötungsentschluss verkündet, ist vom Verhältnis der »Gottessöhne« zu den »Menschentöchtern« die Rede: »Als aber die Menschen sich zu mehren begannen auf der Erde und ihnen Töchter geboren wurden, da sahen die Gottessöhne, wie schön die Töchter der Menschen waren, und nahmen sich zu Frauen, welche sie wollten. [...] $\mathrm{Zu}$ der Zeit und auch später noch, als die Gottessöhne zu den Töchtern der Menschen eingingen und aus ihnen Kinder gebaren, wurden daraus die Riesen auf Erden. Das sind die Helden der Vorzeit, die hochberühmten «, übersetzt Luther ${ }^{39}$, der in einer Fußnote sich beeilt zu versichern, dass die »Gottessöhne « keine »leiblichen Söhne« Gottes seien, sondern lediglich irgendwie zu seinem Gefolge gehörten. Bei den frühen Kirchenvätern und in der jüdischen Tradition werden sie als gefallene Engel angesehen. Jedenfalls kann es dem Gott nicht gefallen, dass die schönen Menschentöchter seinem Umfeld die Köpfe verdrehen und ein titanisches Geschlecht installieren, das auf Sicht seine Herrschaft gefährden könnte. Auch deshalb müssen die schönen Töchter untergehen. In Rincks Vers bekommt ihre Version der biblischen (Vor-)Geschichte der Sintflut auch eine geschlechterkritische Implikation: die »töchter« zählen vorerst nicht zu den »dichtern«, sie sind nur dichter-töchter und gebären statt schlechter Verse riesenhafte Missgeburten. »das ist das ende, beschied gott im himmel«, damit endet die erste Strophe.

Das Gedicht besteht in seiner typografischen Ansicht eindeutig aus drei separaten Strophen unterschiedlicher Länge. Nach der ersten, aus drei Versen bestehenden Strophe, folgt eine kürzere zweite, in der typografisch vor allem die unterschiedli-

\footnotetext{
37 1. Mose 6. 5 f. In: Die Bibel oder die ganze heilige Schrift des Alten und Neuen Testaments nach der Übersetzung Martin Luthers. Stuttgart 1972, S. 20.

38 Rinck, Monika: Die Wahrheit der Dichtung. Rede zur Eröffnung der Festwoche 10 Jahre lyrikline.org. https://lyrikline.wordpress.com/2009/10/29/die-wahrheit-uber-dichtung-rede-von-monika-rinckzur-eroffnung-der-festwoche-10-jahre-lyrikline-org/ (letzter Zugriff: 2.2.2021).

39 1. Mose 6, 1-4. In: Die Bibel oder die ganze heilige Schrift des Alten und Neuen Testaments nach der Übersetzung Martin Luthers. Ebd.
} 
che Länge der beiden Verse ins Auge springt: »und kein fünkchen güte erhellte den pechschwarzen uferwall / die wasser stürzten.« 16 Silben aus Vers 4 stehen nur 5 Silben in Vers 5 gegenüber. Das erhöht die gleichsam lakonische Prägnanz des totbringenden Flutungsgeschehens. Die Wasser stürzen und verschlucken alle weitere Versrede. Metrisch wird dabei der letzte Vers 3 von Strophe 1 (»das ist das ende, beschied gott im Himmel«) an den langen ersten Vers 4 von Strophe 2 angebunden: dem vierhebigen Daktylus, mit dem die erste Strophe endet, folgt in Strophe zwei ein fünfhebiger Daktylus mit Auftakt. Semantisch bedeutsam, weil metrisch akzentuiert, sind bei diesem Versmaß Signalwörter wie »ende« (nicht aber »gott«) in Vers 3, dazu »kein fünkchen güte « und »pech-» in Vers $\overline{4}$, die auf rhythmisch fast anklägerische Weise den strafenden Gott ins Unrecht setzen.

Für die Schriftform des Gedichts ist es nicht unmaßgeblich, dass die sich anschließende vierversige Schlussstrophe in Klammern steht. Die Klammern verändern schlagartig die Tonalität des Gedichts. Satzpragmatisch weisen sie als Zeichen das von ihnen Eingeklammerte sichtbar (nicht hörbar!) als Nebensächliches oder als Ergänzung aus. Hier kann das mit dem Machtgefälle zwischen den Sprechinstanzen zu tun haben. Vorher dekretiert der Gott, und jetzt soll das, was dazu »manch einer sagte «, klingen wie trotzig beiseitegesprochen: »(manch einer sagte sich / bevor ich aus allen tieren / paare bilde und verlade / geh ich lieber unter.)« Die Schlussstrophe formuliert die Selbstbehauptung der vermeintlich schlechten dichter-menschen. In der biblischen Geschichte darf der Gott ja nicht nur den von ihm geschaffenen Menschen vom Erdboden vertilgen. Zugleich hat er einen sehr ausgefeilten Plan parat, wie der von ihm als gerecht und untadelig identifizierte Noah überleben kann. Nachgerade detailversessen und schiffsbaumeisterlich wird vierzehn Bibelverse lang ausführlich entwickelt, welchen Plan der Gott sich für Noah ausgedacht hat (eine Arche) und wie damit der Grundstein für ein neues gottgefälliges Menschengeschlecht (ohne die ganzen riesigen Bastardtitanen) gelegt werden soll, wie der Fortbestand der Tierarten nicht zuletzt deshalb geschützt wird, damit der neue Mensch sie sich untertan machen kann (»Alles Lebendige, das sich regt, soll euch zur Nahrung dienen $\left.\ll^{40}\right)$, wie daraus ein Gottes-Bund mit den Überlebenden entsteht. Von Noah ist in dem Rinck-Gedicht gar keine Rede.

Die unter Todesdrohung selbstbewusste Sprechinstanz des Klammerausdrucks in der letzten Strophe scheint den Archen-Plan des Gottes auf sich zu beziehen, und damit ist sie nicht allein (»manch einer sagte sich:«). Dennoch wird das Überlebensangebot souverän ausgeschlagen. Erzwungene Paarbildung und deren Containerisierung (»und verlade«) zum Zwecke der bloßen Gattungsreproduktion, das scheint keine Option: »geh ich lieber unter)«. Durch das Enjambement zwischen Vers 7 und 8 ([...] allen tieren / paare bilde [...]«) wird plötzlich der Vorgang der Paarbildung freigestellt und metrisch akzentuiert (»paare bilde und verlade), wobei der Rhythmus des Trochäus auch die Assonanz der verladenen Paare hörbar macht. Wer verladen wird und wer zum Verladen bestimmt ist, scheint schon verloren. Hat das Gedicht hier souverän sein Thema gewechselt? Ist zuletzt von Paarbildung (unter Menschentieren) die Rede, solcher, die sich nicht verordnen lässt - um den Preis

40 1. Mose 9.3. In: Neue Jerusalemer Bibel. Einheitsübersetzung. Mit dem Kommentar der Jerusalemer Bibel. Freiburg/Basel/Wien 1985, S. 24. 
des eigenen Untergangs? Von Paarbildung in der Sprache? Von schlechten Reimen? Anyway: »schlechte dichter « jedenfalls ertrinken lieber selbstbestimmt. Mit dieser Behauptungsgeste der Sprechinstanz schließt das Gedicht - fast möchte man sagen: tröstlich.

So in etwa könnte eine kursorische Lektüre der Schriftform von »Alles Sinnen und Trachten« argumentieren. Die Bibelstelle aus der Genesis ist ein vom Wortlaut des Gedichts erzwungener Kontext. Wer sich darüber hinaus für die Autorin und den äußeren Kontext des Gedichts interessiert - innerhalb des Gedichtbandes Helle Verwirrung und überdies als Teil eines Schubers, in dem auch der Band Rincks Ding- und Tierleben enthalten ist, ihr »Doppelalbum« (Christian Metz) von 2009 der könnte auch noch über weitere intertextuelle Bezüge spekulieren: Rinck hat in Berlin bei Klaus Heinrich Religionswissenschaft studiert, der in den 1960er Jahren auch einmal ein Noah-Gedicht geschrieben hat, in dem - wie in »Alles Sinnen und Trachten« - die biblische Geschichte umgedichtet wird: »Aus dem Tagebuch Noah« (darin stellt sich heraus, dass die Taube den Ölzweig aus der »eisernen Ration der Wiederkäuer« auf der Arche geklaut hat: »Sie haben nichts zu fressen ich / werde dich nicht verraten Taube «). ${ }^{41}$ Den Gestus dieser beiden geistesverwandten NoahTravestien könnte man vergleichen.

Klaus Heinrich hat auch während seiner legendären Kolloquien im Seminarraum des Religionswissenschaftlichen Seminars der Freien Universität Berlin - scheinbar digressiv, in Wahrheit aber als eine Konzentrationsübung - beständig Zeichnungen angefertigt, wenn jemand anderes sprach. Häufig Tierzeichnungen. Der Band von Monika Rinck, mit dem der Gedichtband Helle Verwirrung verkoppelt ist -Rincks Ding- und Tierleben - besteht auch aus Zeichnungen der Autorin, häufig Tierzeichnungen, gerne auch Pferdezeichnungen (Monika Rincks erklärtes Lieblingstier, »mein Pferdegang «, wie sie gern kalauert...), denen Kurzprosatexte an die Seite gestellt sind. Die erste Zeichnung/der erste Text dieses Bandes heißt: »Die traurigen Gedanken eines ausrangierten Renners«. Damit korrespondiert er peritextuell mit »Alles Sinnen und Trachten« - dem letzten Gedicht aus dem Doppelband Helle Verwirrung /Rincks Ding- und Tierleben. Sinnen und Trachten wiederum heißt eine Sammlung poetologischer Aufsätze des marxistischen Literaturwissenschaftlers und Übersetzers Hugo Huppert ${ }^{42}$, der vor allem für Majakowski-Übersetzungen berühmt geworden ist und das schönste Pferdegedicht von Wladimir Majakowski ins Deutsche übertragen hat (Rinck ist selber Übersetzerin und zeigt ein emphatisches Interesse für die Bedingungen der Möglichkeit dialogischer Lyrikübersetzungen) es heißt »Gute Behandlung der Pferde « und handelt von einem alten ausrangierten Renner, der auf dem eisigen Kusnjezki Most in Moskau stürzt:

Am Kusnjezki Most / staun sich schon gaffende Mäuler / Die Hosen schlappern ihr Glockenmaul, / Gelächter klimpern / vor Langeweile: / »Ein Gaul ist gestürzt «/ »Gestürzt ist ein Gaul!«/ Der Kusnjezki lachte / Nur ich allein / trat hin / und tat keinen Ton ins Geheule. / Ich schaute / In die Augen des Gauls

\footnotetext{
41 Wiederabgedruckt in: Alfred Brendel - Musik, Sinn und Unsinn. Festschrift anlässlich der Hommage für Alfred Brendel. Hg. v. Konzerthaus Berlin. Berlin 2017, S. 27.

42 Huppert, Hugo: Sinnen und Trachten. Anmerkungen zur Poetologie. Halle/Saale 1973.
} 
hinein... / und kopfstand, umstürzend, die Straßenzeile / Trat hin, sah / Träne um Träne träuft, [...] »Mein Pferd, nicht weinen. / Ich kenne die Beschwerde / Wer sagt Ihnen denn, Sie sei'n weniger wert? / Kindchen, / wir alle sind ein wenig Pferde, / jeder von uns ist auf seine Art Pferd $[\ldots]^{43}$

Eine stärker an Intertexten interessierte Analyse von Rincks Gedicht in seinem Werkkontext könnte den Bezügen zwischen einem Arche Noah-Gedicht am Schluss der Gedichtsammlung und anderen Tiertexten bei Rinck nachgehen oder vielleicht auch solchen zu Pferdegedichten anderer Übersetzer wie dem von Huppert. Das legt die Schriftform von »Alles Sinnen und Trachten« nahe. Was tritt dem an die Seite (im Sinne Middletons), wenn man nun auch eine Lesung der Autorin von »Alles Sinnen und Trachten « analysiert?

\section{$4<<a 11>$ das ist das $\mathrm{EN}: \mathrm{DE}>$ - $\gg$ Live poetry readings are very clearly bounded in time and space. Miss a line and it has gone. $\ll^{44}$}

Die Lyriklesung mit Monika Rinck, aus der hier ein Ausschnitt thematisch sein soll, fand am 3. Dezember 2019 im Siegener Apollo-Theater statt. Das hört sich pompöser an, als es war. Für die Lesung wurde nicht etwa der große Saal (mit 580 Plätzen) geöffnet, sondern das so genannte Siegseiten-Foyer, ein relativ kleiner, schlauchartiger Durchgangsraum, der im ersten Rang des Theaters hinter die Bühne und zu den Schauspielergarderoben führt, aber bisweilen auch für kleinere Veranstaltungen genutzt wird und flexibel bestuhlt werden kann. An diesem Abend kamen etwas über 60 Zuhörerinnen und Zuhörer. Das sind relativ viele für eine Lyriklesung in Siegen. (Gewöhnlicherweise versammeln sich nicht mehr als 20-25 Personen bei Veranstaltungen der Reihe »poetry@ rubens«, die von der Universität kuratiert wird und zweimal jährlich in Kooperation mit dem Theater stattfindet.) Der Grund ist schnell erklärt: die Lesung war zum Pflichttermin eines Universitätsseminars im Wintersemester 2019/20 zum Thema »Living Poetry « erklärt worden, das von Studierenden der Anglistik und der Germanistik gemeinsam besucht wurde. In diesem Seminar wurden Aufzeichnungen von Lyriklesungen und -Performances analysiert. Daran hatten die Studierenden im Laufe des Semesters durchaus großes Interesse gezeigt, ihre Leidenschaft als Lyrik-Rezipierende galt aber in allererster Linie den Gattungen Slam Poetry und Spoken Word. (So erklärt sich, dass viele der Lesungsgäste am 3. Dezember sich sehr wohl als poetry-affin beschreiben würden, mit dem Werk von Monika Rinck aber ganz überwiegend gar nicht vertraut waren.) Von den knapp über 60 BesucherInnen gehörten mehr als 40 diesem Seminar an.

$\mathrm{Zu}$ den Besonderheiten dieses Abends gehörte weiterhin, dass die Lesung ersichtlich sowohl für die Autorin als auch für die neutralen BesucherInnen als Rezeptionsexperiment gerahmt war. Jede/r Besucher fand auf seinem Platz einen Fragebogen vor, mit dem darum gebeten wurde, bestimmte Wahrnehmungen wäh-

\footnotetext{
43 Majakowski, Vladimir: Gute Behandlung der Pferde. Übers. v. Hugo Huppert. In: Ders.: Vers und Hammer. Gedichte und kleine Schriften. Zürich 1959, S. $97 \mathrm{f}$.

44 Middleton: How to Read a Reading, S. 17.
} 
rend der Lesung (oder nach der Lesung) zu notieren (es gab drei distinkte Typen von Fragenbögen, um instruktive Vergleichsgruppen zu bilden...). Die Bedingungen dieses Fragebogenexperiments wurden allen BesucherInnen unmittelbar vor Lesungsbeginn (in Abwesenheit der Autorin) erläutert. Von den knapp über 60 BesucherInnen beteiligten sich immerhin 58 an dem Fragebogenexperiment, damit auch fast alle der nicht-universitären Gäste, was für ein hohes Verpflichtungsgefühl unter den Anwesenden spricht. Als audience hatte sich dieses Publikum i.e.S. bereits vor der Lesung sozial vergemeinschaftet in der Bereitschaft, anders als bei normalen Lesungen auch über ihre Wahrnehmungen bei der Rezeption von auktorial vorgetragener Lyrik partiell Rechenschaft abzulegen. Die Autorin selbst war zuvor um Erlaubnis gebeten worden, diese kleine Rezeptionsstudie durchführen zu dürfen. Sie hatte sehr freundlich eingewilligt, indes war ihr vor allem anfänglich eine gewisse Anspannung anzumerken (Guten Abend, vielen Dank für die Einladung, ich fühl mich n' bisschen geprüft, wenn ich sie alle mit Ihren zetteln hier sitzen sehe ${ }^{45}$, sagte sie zur Begrüßung, nach dem sie vom Moderator eingeführt worden war...), insofern hier das gewöhnliche Ritual einer WasserglasLyriklesung sich zunächst nicht störungsfrei reproduzierte. Es lässt sich also nicht leugnen, dass die Sichtbarkeit der Fragebögen Auswirkungen auf die soziale Praxis selbst hatte, die hier beobachtet werden sollte. Wie so oft, verflüchtigte sich diese initiale Anspannung indes im weiteren Vollzug der Praxis. Deshalb kann man das Arrangement auch als kleines Krisenexperiment im ethnomethodologischen Sinne verstehen: Sichtbar wird u.a., welche Arbeit der InteraktantInnen, welche soziale Leistung Autorin wie Zuhörenden abverlangt wird, damit die impliziten Normen einer gewöhnlichen Wasserglas-Lyriklesung in ihr Recht gesetzt werden.

$\mathrm{Zu}$ den Normen dieses Rituals gehört, dass die Kommunikation zunächst unidirektional erfolgt: eine liest, die anderen hören zu (später dürfen sie dann Fragen stellen oder mitdiskutieren). In aller Regel schreiben die Zuhörenden nicht mit, vielleicht haben sie den Gedichtband, aus dem gelesen wird, auf dem Schoß (aber kaum jemand liest darin mit, denn man möchte die Autorin nicht nur lesen hören, sondern auch lesen sehen.) Für Aufführungssituationen im Allgemeinen - auch wenn sie so wenig theatralisch geprägt sind wie eine Wasserglas-Lyriklesung - gilt, dass eine Differenz zwischen Aufführenden und Zuhörenden klar markiert ist, die zum einen Aufmerksamkeit auf die »Materialität der Stimme « der Vortragenden lenkt und zum anderen das »Verstreichen von Zeit [...] als Bedingung von Wahrnehmung spürbar« macht. ${ }^{46}$

Obwohl sie >nur< zuhören, sind die Zuhörenden konstitutiv für die Lesung ${ }^{47}$ (eine auktoriale Lesung in Abwesenheit von Zuhörenden wäre eher eine Vorbereitungshandlung, ähnlich einer Stimmprobe), mit dem Psychoanalytiker Wilfred R. Bion gesprochen, betreiben die Zuhörenden eine Art containment. Sie sind das Gefäß,

\footnotetext{
45 min. 0.07.10-14 unseres audio-Mitschnitts der Lesung vom 3. Dezember 2019 im Siegener ApolloTheater.

46 Fischer-Lichte, Erika: Grenzgänge und Tauschhandel. Auf dem Wege zu einer performativen.

Kultur. In: Uwe Wirth: Performanz. Zwischen Sprachphilosophie und Kulturwissenschaften. Frankfurt a. M. 2002, S. 277-300, hier: S. 287.

47 Zumthor, Paul: Einführung in die mündliche Dichtung. Berlin 1990, S. 203-216.
} 
in das die Stimme der Autorin ihren Ausdruck hineinlegt, damit spontan Resonanz erzeugt, die bestenfalls in Form einer kommensalen container-contained-Beziehung zurückgegeben und zum Vorteil aller verarbeitet wird. ${ }^{48}$ Bion, der kein Lesungsforscher ist, sondern vielmehr den Gefühlsaustausch in der Kind-Mutter-Dyade beschreibt, stellt sich hierbei ein Tischsituation (commensalis lat. = der Tischgenosse) vor. Übertragen auf die Lyrik-Lesung ist das ein Raum mit Bühne, Stuhl, Tisch, Lampe, Wasserflasche mit Glas, manchmal ein Mikrofon für die Vortragende, dazu eine endliche Anzahl aufnahmebereiter und -williger commensales, meistens auf Stühlen sitzend, bisweilen ein Glas minderwertigen Weißweins auf den Knien balancierend. Monika Rinck selbst, die sich der Bedeutung von Lesungen für die Reproduktion von LyrikerInnen vollauf bewusst ist ${ }^{49}$, beschreibt dieses containment der Zuhörenden auch produktionsästhetisch, gleichsam als kollaborative Arbeit am Text: »Liest man eigene Gedichte je etwas anderes als Korrektur? [...] Am besten: Wenn man sie mit den Augen eines anderen Menschen liest, der einem zuvor mitgeteilt hat, dass sie ihm oder ihr gefielen. Das ist die beste Lektüre - als ein wohlwollender anderer, mit meinen fremden Augen. ${ }^{50}$ Dies im Falle einer positiven Resonanz. Negatives containment, die Scham der Autorin bei Lesungen, bei denen sie eine negative Resonanz empfindet, kann auch dazu führen, dass sie ihre Gedichte umschreibt, erklärt Monika Rinck im Publikumsgespräch im Anschluss an die Siegener Lesung.

Für die Zuhörenden ist die Vergänglichkeit des Klangereignisses Gedichtlesung eine Herausforderung, größer beispielsweise als bei der Musikrezeption. Denn wenngleich es stimmt, was die Philosophin Sybille Krämer über die »Wirkkraft der Stimmlichkeit« schreibt, die nicht »in ihrer Rolle für die sprachliche Semiosis« aufgehe ${ }^{51}$, so fordert der Gedichtvortrag doch auch zur sprachlichen Semiosis heraus. Anja Utler hat das (in dem bislang besten Buch über Lyriklesungen, das mir bekannt ist) als den »Imperativ eines empfundenen Verstehenszwanges « bezeichnet. $^{52}$ Das gehörte Gedicht wird kaum je als reine Wortmusik genossen, sondern auch als bedeutungstragend und »ergänzungsbedürftig « wahrgenommen, als ein später noch $\mathrm{zu}$ lesendes (und wieder und wieder zu lesendes), damit es als »Ganzes« empfunden werde. ${ }^{53}$ Das Hören des Gedichts sei »Verstehensbedingung und Verstehenshinder-

\footnotetext{
48 Bion, Wilfred R.: Attention and interpretation. London 1970, S. 95.

49 In der Münsteraner Poetik-Vorlesung schreibt sie 2015: »Dichterler (Anm. JD: Die Autorin wendet zuweilen das von ihr eingeführte so genannte >türkische Gendering ‘ an, in dem das generische Maskulinum des Deutschen mit Hilfe der Pluralendungen -ler und -lar aus einer Sprache umgebildet wird, die kein grammatisches Geschlecht kennt...) leben nicht vom Buchverkauf. Die Auflagen sind niedrig, die Gewinnspanne für alle Beteiligten gering. Die Lesungen sind das Geschäft. Es geht also - zumindest was das Dispositiv des Finanziellen angeht - weniger darum, Gedichte zu schreiben, als Gedichte zu verkörpern«. In: Monika Rinck: Champagner für die Pferde. Ein Lesebuch. Frankfurt a. M. 2019, S. 321. Später am Abend der Siegener Lesung sagt sie, früher hätten sich die Lyrik-Verleger um das finanzielle Auskommen ihrer AutorInnen gesorgt. Heute sorgen sich die AutorInnen um das finanzielle Auskommen der LyrikVerleger. Seit etwa 2000 gibt es für LyrikerInnen so viel Gelegenheiten, öffentlich zu lesen, wie noch nie zuvor in der Geschichte des literarischen Lebens in Deutschland.

50 Ebd., S. 320.

51 Krämer, Sybille: Negative Semiologie der Stimme. In: Cornelia Epping-Jäger/Erika Linz (Hg.): Medien/ Stimmen. Köln 2003, S. 65-82, hier: S. 73.

52 Utler: »manchmal sehr mitreißend«, S. 26.

53 Ebd.
} 
nis zugleich $\aleph^{54}$, bei Middleton heißt es dazu prägnant: »Miss a line and it has gone, there is no rewind. $\ll^{55}$

Kehrseitig zu dieser Verstehenskomplikation verspricht die auktoriale Gedichtlesung aber auch einen benefit, den die stille Lektüre nicht bereithält: die Anwesenheit der Autorin, die performative Bindung des Gelesenen an Körper und Stimme. Aus der Vorstellung eines impliziten Autor im Rahmen der stillen Lektüre wird bei der Lesung ein Sinn- und Präsenzeffekt durch die Verkörperung des Gelesenen. ${ }^{56}$ Rezeptionstheoretisch kann man das als eine Re-Autorisierung der Lektüre beschreiben: »Die Performanz der Lesung hebt die Trennung zwischen Autor und Werk auf, das bereits Publizierte wird gleichsam aus seiner ersten Zirkulation, dem Umlauf der Schriften und Ideen, wieder in die inszenierte Präsenz des Augenblicks der Entstehung zurückgenommen $\ll^{57}$, so Harun Maye. Die Art, wie die Autorin das Gedicht liest, ihre Körperlichkeit und Stimme werden bedeutungstragend und versprechen auch privilegierte Teilhabe am intendierten Sinn des Gedichts (anders als wenn eine Schauspielerin auf einer Lesebühne fremde Rede einer Dritten interpretierte...). Die philosophische Sonntagsrede zu Sinneffekten diesen Typs steht schon bei Gadamer: »Alles Schriftliche ist [...] eine Art entfremdeter Rede und bedarf der Rückverwandlung der Zeichen in Rede und Sinn. ${ }^{58}$ Manfred Riedel spricht in diesem Zusammenhang von einer »akroamatische[n] Dimension der Hermeneutik «, deren Aufgabe es sei, »das in der schriftlichen Überlieferung Vermittelte [...] in die Unmittelbarkeit des Hörens auf das Sichzusagende zurückzuübersetzen. ${ }^{59}$ Um wieviel verbindlicher (autoritärer?) erscheint dieser Sinneffekt überdies, wenn die Schrift von ihrer Autorin selbst in Rede zurückverwandelt und verkörpert wird? Indem die Autorin für die in ihrem Namen versammelten Zuhörenden einen Hörraum errichtet, beglaubigt sie ihre Sprecherposition. Middleton bringt diese Koppelung von Autor/ Werk in der Lesung auf den Punkt: »The author performs authorship by reading her or his poetry. $\ll^{60}$

Warum nun bestehen die allerwenigsten Lyriklesungen nur aus Lyriklesung? Warum übersteigt der Anteil an Rahmungskommunikation (Begrüßung, Moderation, performative Epitexte, Autorengespräch, Publikumsgespräch) zeitlich den des reinen Werkvortrags beträchtlich? Offenbar ist die »Dissonanz«, von der Anja Utler spricht, »zwischen der tatsächlichen körperlichen Anwesenheit der Autorin und ihrer instabilen kommunikativen Bindung an eine narrativ kaum rezipierbare und

\footnotetext{
54 Ebd., S. 6.

55 Middleton: How to Read a Reading, S. 17.

56 Vgl. Utler: »manchmal sehr mitreißend«, S. $27 \mathrm{ff}$.

57 Maye: Vortrag/Lesung, S. 341.

58 Gadamer, Hans-Georg: Wahrheit und Methode. Grundzüge einer philosophischen Hermeneutik. Tübingen ${ }^{2} 1965$, S. 371.

59 Riedel, Manfred: Hören auf die Sprache. Die akroamatische Dimension der Hermeneutik. Frankfurt am Main 1990, S. 176. Vgl. dazu auch Jäger, Ludwig: Audioliteralität: zur akroamatischen Dimension des Literalen. In: Natalie Binczek/Uwe Wirth (Hg.): Handbuch Literatur \& Audiokultur. Berlin/New York 2020, S. 61-84.

60 Middleton: How to Read a Reading, S. 22.

61 Utler: «manchmal sehr mitreißend«, S. 21.
} 
auch nicht als alltägliches Selbstgespräch klassifizierbare $>$ Ich $<-R e d e ~ «{ }^{61}$, worin die Lesung von Gedichten besteht, besser auszuhalten, wenn sie von Rahmungskommunikation - insbesondere solcher der Autorin - eingehegt wird. Für die Praxis der zeitgenössischen Lyriklesung scheint sie konstitutiv zu sein. Deshalb verdienen insbesondere die performativen Epitexte aus dem Mund der Autorin nicht als bloßer Kontext der Lyriklesung angesehen zu werden, sondern Gedichtvortrag wie performative Epitexte gehören zu einem Ensemble kommunikativer Gattungen, aus denen die soziale Praxis Lyriklesung sich zusammensetzt. Das kann man aus der linguistischen Gesprächsforschung lernen. ${ }^{62}$

Gesprächsförmig ist die Lyriklesung - wie wir gesehen haben - allemal, auch wenn die Redeanteile nicht gleichmäßig verteilt sind. Die BesucherInnen einer Lesung willigen von vornherein in die Rolle ein, primär als hearer an dem Gespräch teilzunehmen, das die Autorin mit ihnen führt. Konstitutiv für Gespräche ist weiterhin, dass »die Teilnehmer [...] ihre Äußerungen spezifisch auf den jeweiligen Äußerungsadressaten und dessen Vorwissen zuschneiden. « ${ }^{63}$ In der Ethnomethodologie wird hier von »Recipient Design« gesprochen ${ }^{64}$ Der performative Epitext als kommunikative Gattung vor oder nach einer Gedichtlesung würde - einem Axiom der linguistischen Gesprächsforschung zufolge - dazu dienen, ein Verstehen mitzuermöglichen (bzw. der Entstehung von Verständigungsproblemen vorzubeugen). In diesem Sinne könnte man die performativen Epitexte daraufhin befragen, was sie dem Verständnis des Gehörten/zu Hörenden in actu hinzufügen. Fungieren Sie als Verständnishilfe, als Hör-Vorschrift oder -Angebot, als Hörmotivation, in welcher Weise leiten Sie die Rezeption des Gedichtes an? Sind Sie eine Antwort der (Gesprächs-)Praxis selbst auf die Ergänzungsbedürftigkeit der merkwürdigen »IchRede« (Anja Utler), aus der eine Lyriklesung besteht?

»Alles Sinnen und Trachten« las Monika Rinck ganz am Schluss des von ihr programmierten Teils der Siegener Lesung (danach erfolgte ein sehr langes Publikumsgespräch, in dessen Verlauf noch ein weiterer Gedichtwunsch geäußert wurde, den sie erfüllte...). Dramaturgisch scheint dieses Gedicht aus Sicht der Autorin besonders gut geeignet, die Praxis, die eine Lyriklesung darstellt, zu beschließen. Vielleicht weil es eingängiger ist als viele andere Gedichte Rincks, angetan dazu, einen Prägnanzpunkt zu setzen (dem nicht noch weitere performative Epitexte nachfolgen müssen) - ein >Rausschmeißer <, der die Zuhörenden mit einem positiven Rezeptionserlebnis entlässt. Strukturell entspricht die Platzierung dieses Gedichts am Ende der Lesung auch der im Gedichtband Helle Verwirrung, in dem es zuerst erschien.

Zugegeben: Nicht alle Aspekte der Liveness, der »Body Communication ${ }^{65}$ dieses Lesungsereignisses sind aufzeichnungsfähig (auch dann nicht, wenn von der Monika Rinck-Lesung nicht wie hier ein Audio-, sondern ein perfekter Videomitschnitt

\footnotetext{
62 Vgl. Goodwin, Charles: Conversational Organization: Interaction between Speakers and Hearers. New York 1981; Jörg Bergmann: Ethnomethodologische Konversationsanalyse. In: Ludger Hoffmann (Hg.): Sprachwissenschaft. Ein Reader. Berlin/New York 2010, S. 258-274.

63 Bergmann: Ethnomethodologische Konversationsanalyse, S. $270 \mathrm{f}$.

64 Ebd., S. 271.

65 Vgl. Novak, Julia: Live Poetry. An Integrated Approach to Poetry in Performance. Amsterdam/New York 2011, S. 145-171.
} 
angefertigt worden wäre...) Das betonen völlig zu Recht alle LesungsforscherInnen von Middleton bis zu den Performance Studies. ${ }^{66}$ Warum aber sollte man nicht - just for a start - eine Transkription in Anlehnung an die Standards der linguistischen Gesprächsforschung versuchen? Auch den GesprächsforscherInnen ist völlig klar, dass ihre Konventionen zur Aufzeichnung gesprochener Sprache nicht alle Aspekte dieser Praxis erfassen. Wenigstens einige aber ganz unbestritten, und deshalb könnte eine empirisch gehaltvolle Lesungsforschung - eine, die ihre Ergebnisse überprüfbar zu machen versucht - sich an diesen Konventionen orientieren.

Apollo Theater Siegen (poetry@ rubens) 03.12.2019 -

Lesung »Alles Sinnen und Trachten« (Gedicht und Epitext)

$0.45: 57-0.46: 42^{67}$

MR - Monika Rinck

P - Publikum

\begin{tabular}{|c|c|c|c|}
\hline $45: 57$ & 1 & MR & $\begin{array}{l}\text { und das geDICHT was so'n bißchen (.) ist wie_äm (.) } \\
\text { der LETZte spruch der yogalehrerin }\end{array}$ \\
\hline $46: 04$ & 2 & & du hast jeden muskel maximal entspannt und gedehnt \\
\hline $46: 08$ & 3 & & du hast alles getan du musst nichts mehr tun \\
\hline $46: 09$ & 4 & & ${ }^{\circ} \mathrm{hh}$ \\
\hline $46: 11$ & 5 & & ALLes sinnen und trachten \\
\hline $46: 13$ & 6 & & $(2.5)$ \\
\hline $46: 15$ & 7 & & $\begin{array}{l}\text { die MENschen waren !SCHLECHT!e dichter (-) und ihre } \\
\text { TÖCHter trugen riesige BAStarde aus }\end{array}$ \\
\hline $46: 20$ & 8 & & $\begin{array}{l}\text { das ist das EN:DE (-) beschied GOTT im himmel (.) und } \\
\text { kein FÜNKchen GÜte }\end{array}$ \\
\hline $46: 27$ & 9 & & $\begin{array}{l}\text { erhellte den pech:schwarzen uferwall die wasser } \\
\text { !STÜRCHZ!ten }\end{array}$ \\
\hline $46: 31$ & 10 & & ${ }^{\circ}$ hhh \\
\hline $46: 33$ & 11 & & $\begin{array}{l}\text { manch einer sagte sich bevor ICH aus allen tieren } \\
\text { PAAre bilde (.) und verLA:DE }\end{array}$ \\
\hline $46: 39$ & 12 & & geh ich lieber unter \\
\hline $46: 41$ & 13 & & $<<:-)>$ dankeschön $>$ \\
\hline $46: 42$ & 14 & $\mathbf{P}$ & {$[((\operatorname{lach} t))]$} \\
\hline $46: 42$ & 15 & & {$[((k \operatorname{latsch} t))]$} \\
\hline
\end{tabular}

Weil wir das Gedicht schon gut kennen, macht es uns wenig Mühe zu erkennen, wo es hier anfängt und aufhört. Für das gesprächsanalytische Transkript ist die (Wert-)Unterscheidung von Gedicht und Epitext gerade irrelevant. Gemäß dem »Natürlichkeitsprinzip« sollen die Daten jener Praxis, über die »anhand der Untersuchungsmaterialien Aussagen gemacht werden sollen«, völlig neutral, unselektiv, gleichsam »ökologisch« erhoben werden. ${ }^{68}$ Hier nun sieht man, dass die Autorin den Beginn ihres Gedichtes durchaus durch ein hörbares Einatmen (von ca. 0.5-0.8 Sek. Dauer) markiert.

Voraus geht eine einleitende Bemerkung, die das Gedicht mit einem spruch der Yogalehrerin vergleicht, mit dem, was die Yogalehrerin am Schluss der Stunde zu

\footnotetext{
66 Vgl. Benthien, Claudia/Prange, Catrin: Spoken-Word-Literatur und Poetry Slam.

67 Die Transkription erfolgt nach dem Basistranskript-Standard des Gesprächsanalytischen Transkriptionssystem GAT 2. In: Selting, Margret et al.: Gesprächsforschung - Online-Zeitschrift zur verbalen Interaktion 10 (2009), S. 353-402 (www.gespraechsforschung-ozs.de). Vielen Dank an Manuel Kleffner für die Mitarbeit bei der Erstellung dieses Transkripts.
}

68 Deppermann, Arnulf: Gespräche analysieren. Eine Einführung. Wiesbaden ${ }^{4} 2008$, S. 25. 
ihren ElevInnen sagt, ein resümierendes Lob für die unternommenen körperlichen Anstrengungen, eine Bilanz, die den Sinn und Erfolg der vorausgegangenen Körperarbeit bescheinigt (du hast jeden muskel maximal entspannt und gedehnt) und damit die ElevInnen wieder in ein wohlverdientes Jenseits der Anstrengung entlässt (du hast alles getan du musst nichts mehr tun), das als Endentspannung gleichwohl noch Teil der Yoga-Praxis selbst ist. Wenn Monika Rinck ihr Schlussgedicht mit dieser Bemerkung aus dem Kontext motivierender Bewertungskommunikation in Beziehung setzt, dann wird aus ihr die Yogalehrerin, die den Zuhörenden Respekt zollt für die anstrengende Hörarbeit während dieser Lesung und das Schlussgedicht gleichsam als Dank und Entlastung für vorangegangene »Verständnishindernisse « (Utler) entbietet (du musst nichts mehr tun als nur noch einmal zuzuhören - diesmal aber völlig tiefenentspannt und ohne hermeneutische Komplikationen, ohne »Ergänzungsbedürftigkeit« nicht-alltäglicher »Ich-Rede«).

Rhetorisch ist dieser Epitext also eine Art captatio benevolentiae. Damit wird einerseits eine Gedichtgabe angekündigt, mit deren Hilfe sich die Rednerin abschließend des Wohlwollens möglicherweise skeptischer ZuhörerInnen zu versichern versucht. Andererseits ist damit ein Versprechen gegeben, dass im Zustand der totalen, ganzkörperlichen Entspannung (nach der anstrengenden Hör-Arbeit) ein entlastendes After-Hour-oder Chill-out-Gefühl aufkommen könnte, das auch ein neues, anderes Verstehen ins Werk setzt. Dieser performative Epitext gehört jedenfalls eindeutig der Kategorie Hörmotivation zu. Wie immer bei solchen Versprechungen geht die Sprecherin damit auch das Risiko einer »Folgeerwartung ${ }^{69}$ ein: was, wenn die Zuhörenden das Abschlussgedicht gar nicht als entlastend empfinden, sondern als Fortsetzung der erschöpfenden Hör-Muskeldehnung...? (Das Lachen, der Applaus der Zuhörenden nach der Schlusspointe von »Alles Sinnen und Trachten « - der Selbstermächtigung der totgeweihten menschen-dichter geh ich lieber unter spricht dafür, dass die Lesung tatsächlich mit einem positiven containment endet. Oder ist es die Erleichterung darüber, dass die Lesung jetzt wirklich vorbei ist...?)

Was den performativen Epitext für dieses Schlussgedicht geeignet erscheinen lässt, ist die gleichsam verdoppelte Selbstreferenz: Monika Rinck wird nicht nur mit der Yoga-Lehrerin identifiziert, die ihre ElevInnen in den Abend entlässt, sondern instantan auch mit jenen Menschen des ersten Gedichtverses, von denen gesagt wird, sie seien »schlechte dichter«. In der besonderen Autor-Werk-Koppelung der Lesungssituation sind die Zuhörenden nun disponiert, das Verdammungsurteil des zürnenden Gedicht-Gottes nicht zuletzt auch auf die Sünden dieser Autorin zu beziehen, der man gerade 47 Minuten lang gelauscht hat. Selbstironisch wird das Weltgericht der Noah-Geschichte zu einem Urteil über die Performanz der Autorin. Das ist eine Lesart, die man auch schon an der Schriftform des Textes hätte entwickeln können (vor allem wenn man seine Stellung am Ende des Gedichtbandes mitberücksichtigt), unabweisbar wird diese Deutung erst im Vollzug von performativem Epitext und der auktorialen Verkörperung des Gedichts im Kontext einer Lyriklesung. Amplifiziert wird damit auch die Selbstbehauptung der Dichtersünder in der Schlussstrophe. Die Autorin selbst ist mitgemeint und führt sich als Gemeinte

69 Ebd., S. 68-70. 
vor, wenn sie den Untergang trotzig der Unterwerfung unter ein göttlich-paternalistisches Paarbildungsdekret vorzieht.

Die Klammer, mit der in der Schriftform die letzte Strophe des Gedichts ausgezeichnet ist, kann Monika Rinck im Vortrag nicht unmittelbar hörbar machen. Sie versucht sie zu markieren durch eine Atempause von annähernd $1 \mathrm{sec}$. Dauer, die fast einer Zäsur gleichkommt und damit die Zuhörenden für die veränderte semantische wie gestische Tonalität der Schlussstrophe disponiert.

Mit Blick auf die Intonationseinheiten, die das Transkript kenntlich macht, fällt des Weiteren auf, dass orate und literate Gedichtstrukturen keinesfalls deckungsgleich sind. ${ }^{70}$ Den Vers 1 (Jambus) liest Monika Rinck rhythmisch ganz im Sinne des Metrums der Textvorlage, wobei die dritte Hebung in Vers 1 (!schlechte! dichter) aus semantischen Gründen einen starken Fokusakzent erhält. Fast parodistisch klingt die ironische Selbstbezichtigung. Schon in Vers 3 aber betont die Vortragende nicht mehr durchgängig daktylisch, sondern dehnt den Akzent auf der ersten Silbe von EN:DE lustvoll auf die unbetonte zweite Silbe aus. Durch diese schwebende Betonung entsteht eine Zäsur, die der Autorin ermöglicht, den Restvers unmetrisch zu lesen und ganz nach semantischem Belieben zu akzentuieren. Hier ist das EN:DE der bestehenden metrischen Ordnung von Strophe 1 und 2 erreicht. Auf fast plakative Weise betont die Vortragende GотT, das Machtagens dieses Verses, das in der Schriftform noch in die Senkungen des Versfußes eingelassen war (dadurch subtil bedrohlicher wirkte?): »das ist das ende, beschied gott im himmel.« Die Prosodie des Vortrags geht hier demgegenüber ganz auf Verständlichkeit.

Besonders auffällig ist die Diskrepanz zwischen den Verseinheiten der Schriftform und den Intonationseinheiten des Vortrags in Strophe 2. Die Versgrenze zwischen V.4 und V.5 (»und kein fünckchen güte erhellte den pechschwarzen uferwall. / die wasser stürzten «) macht Monika Rinck unhörbar, der Umbruch wird ganz der dramatischen Steigerung des Sprechtempos und der Sprechintensität geopfert, in welcher der Vortrag auf den starken Fokusakzent von ! STÜRCHZ! ten zuläuft. Dabei bemüht die Autorin sogar eine dialektale Klangfarbe, um den stürzenden Sintflutwassern noch mehr parodistisches Ausdrucksgewicht zu verleihen, etwas das man als Bühnen-Rheinisch bezeichnen könnte (so wie in den 1990er Jahren in der TVlate night show »RTL Samstag Nacht « Comedians in grotesk gemusterten Moderatoren-Pullovern immer die »Sporcht-Schau « veralberten...). Ein Effekt dieses verschluckten Versumbruchs und dem Extra-Akzent auf ! STÜRCHZ!ten ist überdies, dass die Autorin den unreinen Binnenreim mit kein FüNKchen Güte aus Vers 4 hörbar machen kann, den man beim Lesen kaum privilegiert hätte, weil die Strophe insgesamt so reich ist an Anklangphänomenen (»und kein fünkchen güte erhellte den pechschwarzen uferwall. / die wasser stürzten. «). Die Betonung des ü-Klang im Vortrag dient zur Diskreditierung des Gottes, der ohne den letzten diminutiv-glimmenden Rest Güte seine Sintflut herabstürzen lässt. Auch hier setzt die Prosodie vor allem auf Verständlichkeit.

\footnotetext{
70 Vgl. Boneß, Antje/Olfert, Helena: Gegenüberstellung einer mündlichen und einer schriftlichen Textversion: Orate und literate Strukturen. In: Utz Maas (Hg.): Orat und literat. Grazer Linguistische Studien (GLS) 73 (2010), S. 169-181.
} 
Eine letzte Beobachtung noch zum insgesamt sehr lakonischen Vortrag von Strophe 3, der auch tonlich im starken Gegensatz zum alttestamentarischen Bühnenrheinisch von Strophe 2 stehen soll: Monika Rinck betont vor allem das ICH in Vers 7, das den selbstreferentiellen Zug des Gedichts noch unterstreicht: zu den »manch eine[n] , die trotzig selbstbestimmt in den Untergang gingen, kann auch und nicht zuletzt das lyrische ICH gezählt werden, das hier vorträgt. Wie zum klanglichen Beweis seines Dichtertums, betont dieses Vortrags-Ich sehr prononciert und gedehnt (wie die Muskeln beim Yoga) den Binnenreim in Vers 8 PAAre bilde (.) und verLA:DE. Danach muss wirklich kaum mehr etwas getan werden: den Schlussvers geh ich lieber unter liest die Autorin ganz prosaisch, ein echter Rausschmeißer. Zur Markierung des Gedichtendes erfolgt ein mimisch-stimmlicher Registerwechsel: $<<$ : - ) steht für die »smile voice «, in die auch die Erleichterung über das Ende der Performanz eingeschrieben ist. Der Gedichtvortrag von »Alles Sinnen und Trachten« besteht aus zwei gänzlich differenten Tonlagen, mit denen asymmetrische Sprecherpositionen markiert sind: für die Strophen 1 und 2 der (parodierte) hohe Ton des zürnenden Gottes, in der Schlussstrophe die mutige Dichterler-Gegenrede aus dem Parkett. Damit führt die Performanz praktisch vor, worüber Monika Rinck in ihrer Göttinger Poetik-Vorlesung 2019 schon räsonniert hatte (auch mit Blick auf noch viel komplexere Gedichte als »Alles Sinnen und Trachten«):

»Eine genaue Lektüre vermag ein Gedicht, selbst da, wo es weder Rollen noch Personen auszeichnet, in mehrere Stimmen aufzufächern. Einen Anhaltspunkt kann dabei auch die Autorenlesung geben. Spricht der Autor, spricht die Autorin mit unterschiedlichen Stimmen? Suggeriert seine/ihre gestische Rede einen Dialog oder Polylog? Höre ich die gedankliche Rede und Gegenrede, die Einzelschritte der Selbstverbesserung auf dem Weg zur Erkenntnis in Form eines Gesprächs oder Selbstgesprächs? Das ist häufig der Fall, meine ich. Das Gedicht, als Sprachkunstwerk, kann zuweilen einzelne Zwischenschritte einfach stehenlassen, so als würde die Sprachkorrektur materiell. $\ll^{71}$

\section{Schluss: Lesungsboom und Alltagshermeneutik}

Empirisch verdient die hier vorgelegte Untersuchung genannt zu werden, weil sie (anders als die historische Lesungsforschung) eine konkrete zeitgenössische Lesungsperformanz dokumentiert und ein vor Publikum vorgetragenes Gedicht nicht zuletzt mit den Mitteln der linguistischen Gesprächsforschung zu analysieren versucht. Diese Lektüre eines Vortragsereignisses (das erst in Transkriptionstext verwandelt werden musste, um analytisch zugänglich zu werden), wird der traditionell schrifthermeneutischen Lektüre des Gedichtes in der Buchpublikation an die Seite gestellt. In welchem Verhältnis stehen diese beiden Lektüren zueinander? Bedürfen sie ihrer wechselseitig? Ich denke, man muss nicht so weit gehen wie der sympathische britische Lyrikpädagoge Middleton und behaupten, beide Lektüren - das »silent reading « des Gedichts auf der Buchseite und die ästhetische Hör-Erfahrung

71 Rinck, Monika: Wirksame Fiktionen. Göttingen 2019, S. 26 f. 
seiner »oral performance « im Rahmen einer Lyriklesung - seien für sich genommen jeweils defizitär, »incomplete«. Wie zu sehen war, kommen beide Lektüren auch gut ohne einander aus: Man braucht keine Luther-Bibel zur Hand, um als Lesungsbesucher in »Alles Sinnen und Trachten« den Tonwechsel in der dritten Strophe mitzuvollziehen, und man muss als Gedicht-Leserin nicht den Bühnenakzent von Monika Rinck (! schlechTe! dichter) im Ohr haben, um die Selbstreferenz dieser Noah-Travestie zu verstehen. Dennoch ist es instruktiv, beide Lektüren aufeinander zu beziehen - gerade vor dem Hintergrund der rezenten Transformationen des literarischen Lebens, dem Lesungsboom im deutschsprachigen Raum seit ungefähr 2000, der etwa zeitgleich verläuft zu der erstaunlichen Blütezeit der zeitgenössischen deutschsprachigen Lyrik, die Christian Metz so eindrücklich beschrieben hat. Die Bereitschaft zum silent reading eines Gedichts auf einer Buchseite scheint abzunehmen, dagegen besuchen erstaunlich viele Menschen Lesungsveranstaltungen, um sich der ästhetischen Erfahrung auszusetzen, ein ihnen vollständig unbekanntes Gedicht, eine »narrativ kaum rezipierbare «, merkwürdige »Ich-Rede« (Utler) einer Dichterstimme in Gegenwart anderer zu rezipieren. Wenn dem so ist, dann werden Performanz-Lektüren wie die hier vorgeführte umso relevanter - womöglich gerade ohne hermeneutische Rückversicherung an der Schriftform des Textes.

Eine weitere Konsequenz dieses Lesungsbooms bei abnehmender Lektürebereitschaft ist literaturtheoretischer Art: Gestärkt wird unzweifelhaft die Koppelung von Autor und Werk. Ohne die Autorin, die es in actu verkörperte, gäbe es - überspitzt gesagt - gar keine ästhetische Erfahrung des Werks »Alles Sinnen und Trachten «. Umso wichtiger scheint der subjektiv gemeinte Sinn, der sich im auktorialen Vortrag (und in den ihn umrahmenden performativen Epitexten) vermeintlich ausstellt. Aus der bildungsbürgerlichen Schrifthermeneutik (einer vor- oder nachbereitenden Gedichtlektüre im Buch als Primäranreiz des Lesungsbesuches) wird gleichsam die Alltagshermeneutik unserer Lektüren von Gesichtern, Körpern, Stimmen beim präsenzkulturellen Vortragsereignis. ${ }^{72}$ Monika Rinck ist sich der Bedeutung dieser veränderten Gedichtrezeption vollkommen bewusst: »Ich höre den Dichter, die Dichterin einatmen, und dann höre ich das, was darauf folgt. Wir erinnern uns an eine der Grundannahmen menschlicher Kommunikation: Ich weiß nicht, was im Kopf des anderen vor sich geht. Aber ich sehe sein Gesicht. Ich bin angehalten zur Deutung. Peter von Matt vergleicht das Gedicht mit seinem deutschen Reimwort: dem Gesicht. Wir haben es mit einer begrenzten Fläche zu tun, bei unendlich sprechendem Inhalt. «73 Auf einem der Fragebögen zur Monika Rinck-Lesung 2019 in Siegen hatten wir die Frage gestellt: »Was würden (oder werden) Sie einem Freund, einer Freundin von dieser Lesung erzählen?« Aus den Antworten: »Interessante Erfahrung; andere, intensivere Wahrnehmung, wenn man die Autorin persönlich kennenlernt, hört, was sie zu ihrer Arbeit sagt.«»Über die sehr einzigartige Vortragsart von

\footnotetext{
72 »Der Autor, der sein Buch öffentlich verkörpert, anstatt reglos daraus vorzutragen, wird zum Kunstereignis, das Buch zu dessen Kommentar [...]«, schreibt schon zu Beginn des Lesungsbooms um 2000 die Kritikerin und Autorin Ursula März. S. dies.: Rauchen und Rucken. Die Dichterlesung als Ereignis und Problem. In: Frankfurter Rundschau v. 26. Februar 2002. Vgl. dazu auch: Thomas Wegmann: Zwischen Gottesdienst und Rummelplatz. Das Literaturfestival als Teil der Eventkultur. In: Ders./Erhard Schütz: literatur.com. Tendenzen im Literaturmarketing. Berlin 2002, S. 121-136.
}

73 Rinck, Monika: Die Wahrheit der Dichtung (letzter Zugriff: 2.2.2021). 
Monika Rinck. Ohne sie hätten ihre Texte eine komplett andere Wirkung auf mich gehabt.«»Dass man ihrer Meinung nach nicht verstehen muss, um die Gedichte zu genießen.«

Funding Open Access funding enabled and organized by Projekt DEAL.

Open Access Dieser Artikel wird unter der Creative Commons Namensnennung 4.0 International Lizenz veröffentlicht, welche die Nutzung, Vervielfältigung, Bearbeitung, Verbreitung und Wiedergabe in jeglichem Medium und Format erlaubt, sofern Sie den/die ursprünglichen Autor(en) und die Quelle ordnungsgemäß nennen, einen Link zur Creative Commons Lizenz beifügen und angeben, ob Änderungen vorgenommen wurden.

Die in diesem Artikel enthaltenen Bilder und sonstiges Drittmaterial unterliegen ebenfalls der genannten Creative Commons Lizenz, sofern sich aus der Abbildungslegende nichts anderes ergibt. Sofern das betreffende Material nicht unter der genannten Creative Commons Lizenz steht und die betreffende Handlung nicht nach gesetzlichen Vorschriften erlaubt ist, ist für die oben aufgeführten Weiterverwendungen des Materials die Einwilligung des jeweiligen Rechteinhabers einzuholen.

Weitere Details zur Lizenz entnehmen Sie bitte der Lizenzinformation auf http://creativecommons.org/ licenses/by/4.0/deed.de. 